\title{
Force Control in Monopod Hopping Robot While Landing
}

\author{
Vaidyabhushan Leela Krishnan, Pushparaj Mani Pathak, Satish Chandra Jain \\ Department of Mechanical and Industrial Engineering, Indian Institute of Technology, Roorkee, India \\ E-mail: \{vlk08dme, pushpfme, sjainfme\}@iitr.ernet.in \\ Received August 23, 2010; revised September 12, 2010; accepted September 28, 2010
}

\begin{abstract}
In this paper, the issue of control of impact forces generated during the interaction between the hopping robot toe and the ground while landing has been considered. The force thus generated can damage the robot altogether. With the objective to control these impact forces, impedance control strategy has been applied to the hopping robot system. The dynamics pertaining to the impact between robot toe and ground has been modeled as in case of a ball bouncing on the ground. Bond Graph theory has been used for the modeling of the hopping robot system. Simulation results show that impact forces generated during the landing has been controlled to a specified limiting value. This model and the corresponding analysis can be further extended for understanding the dynamics involved in continuous hopping of robot with constant height and velocity control.
\end{abstract}

Keywords: Hopping Robot, Impact Forces, Impedance Control

\section{Introduction}

In recent years, legged robots, especially biped robots, have been developed to the extent that human-like walking has become possible. In the next stage, robots need to move faster and get over larger obstacles. In this respect, hopping robots offers a potential solution. Due to the possibility of adjusting the stride length irrespective of the structural limits of a hopping robot, it can move faster and avoid larger obstacles than walking. Hopping robots can move with greater dexterity in an environment characterized by holes, steps and bumps. But an important issue related to hopping robot locomotion is reducing the impact force from the ground at the instant of robot landing which may, otherwise, cause damage to robot.

In order to resolve this problem, Raibert [1] used hydraulic cylinders and Hyon et al. [2] used mechanical springs in their robotic legs. However, Hydraulic cylinders don't have enough control performance, especially in the edge of the cylinders. Considering hopping as an extended function of walking, use of mechanical springs makes the hopping robot highly dependent on spring characteristics and the control to be complicated. Hence, suppressing impact force in the landing phase without cylinders or mechanical springs is a big issue to be dealt with. In addition to the force control during landing, it is also important to achieve a desired position of center of gravity (CG) of the hopping robot at the bottom most point i.e. bottom of stance phase. This ensures good trajectory robustness during the next hop.

In order to deal with these issues, Sato et al. [3] has used a combined method of soft landing trajectory of robot body and optimal approach velocity to the ground. Fujii and Ohnishi [4] investigated this issue further and proposed a smooth transition method from compliance control to position control. However these methods could not achieve the desired objective of constant force control during the landing phase and precise position control at the bottom.

In this paper the issue under consideration is dealt with by controlling the driving point stiffness (impedance) at the interaction port between hopping robot toe and the physical ground i.e. environment. Pathak et al. [5] have used this control strategy employing passive degree of freedom (DOF) in controller domain for the control of interaction forces between space robot tip and environment. The proposed controller deals adequately with the issue of force (compliance) control i.e. reducing ground impact forces at touchdown and position control at bottom so as to prepare hopping robot for the next hop. Bond Graph theory [6] has been used for the modeling of the hopping robot system. Simulations have been performed using SYMBOLS Shakti [7], a bond graph modeling software.

The paper is organized as follows. Section 2 presents 
the bond graph modeling technique. Section 3 presents the modeling of the impact of hopping robot toe with ground as in case of a ball bouncing on ground. Section 4 presents the dynamic modeling of a hopping robot. Section 5 describes the impedance control scheme being used to control the hopping robot and presents the corresponding simulation results. Section 6 discusses the results and proposes the future work.

\section{Bond Graph Modeling Technique}

The bond graph technique offers a very powerful tool for modeling physical systems and formulating the system equations. Systems from diverse branches of engineering science can be modeled in a unified manner using bond graphs [6].

The underlying idea in bond graph modeling is that physical systems in various domains interact dynamically through power as the common currency of exchange. Hence bond graphs, essentially represents the power exchange portrait of the system. Power is expressed as multiplication of two factors viz. generalized effort and generalized flow.

In bond graph modeling, a system is considered to be a dynamic unit constituted of inertances (I), compliances (C), and dissipators (R). The external source inputs to system are expressed as source of effort (SE) or source of flow (SF) elements. Two multi-port elements transformer (TF) and gyrator (GY) are also used. TF element performs flow to flow or effort to effort conversion whereas GY element converts flow to effort or effort to flow. System Constraints are represented using ' 1 ' junction (representing constant flow) and ' 0 ' junction (representing constant effort) elements. The elements are connected by line segments called bonds. The bonds portray the path of exchange of power within the constraint structure and elements. Power direction assignment in a bond graph is arbitrary in nature and may be compared with the fixing of coordinate systems in the corresponding physical domain. The notion of causality provides a tool for formulation of system equations. The notion of causality also enables a modeler to perform qualitative analysis of system behavior, viz. controllability, observability, fault diagnosis, etc. Thus Bond graph modeling technique enhances a modeler's insight into physical system behavior.

Figure 1 shows the various elements of bond graph along with their respective constitutive laws. In the present work, bond graph Modeling and its simulation are performed using SYMBOLS2000 ${ }^{\circledR}$ [7]. It runs on windows XP environment.

\section{Modeling of Impact between Hopping Robot Toe and Ground}

This section presents the modeling of impact dynamics of a hopping robot toe with ground. The modeling of the phenomenon is inspired from the dynamics of a ball hitting the ground [8]. Figure 2 shows a schematic figure representing the impact of a ball with ground. The $\mathrm{Y}$-axis of the absolute (inertial) reference frame $\{A\}$ shown in the figure represents the direction of vertical motion of the ball. In the figure, $y_{B}$ and $y_{G}$ represents the displacement of ball and ground with respect to the frame $\{A\}$. Similarly $V_{B}, V_{G}$ denote the velocities of the ball and the ground respectively with respect to the inertial reference frame.

The velocity of the ball and the ground can be derived by considering their kinematics relationships as:

$$
\begin{aligned}
& V_{B}=d y_{B} / d t, \\
& V_{G}=d y_{G} / d t .
\end{aligned}
$$

Hence $V_{r}$, relative velocity of ball with respect to the ground can be written as,

$$
V_{r}=V_{B}-V_{g}
$$

Hence, ' $y_{r}$ ' relative displacement of the ball with respect to ground or specifically the point of contact is represented as,

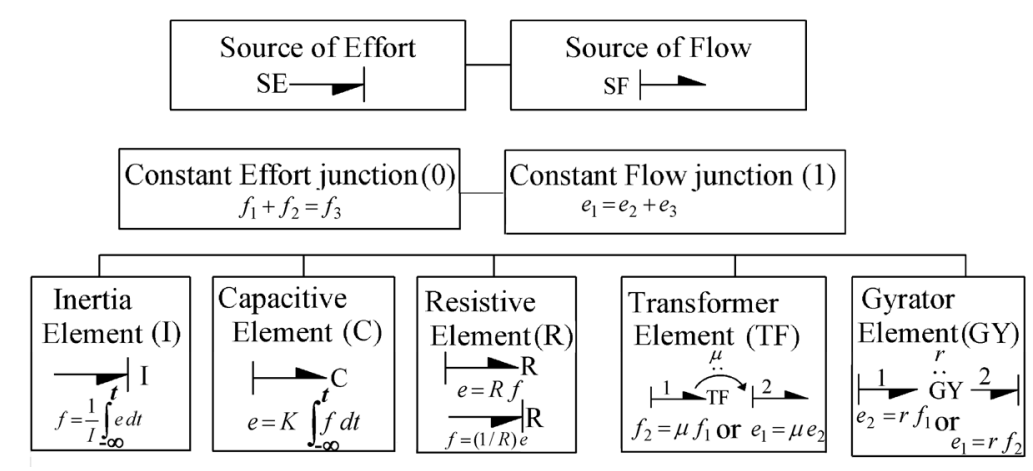

Figure 1. Bond graph elements and their constitutive laws. 


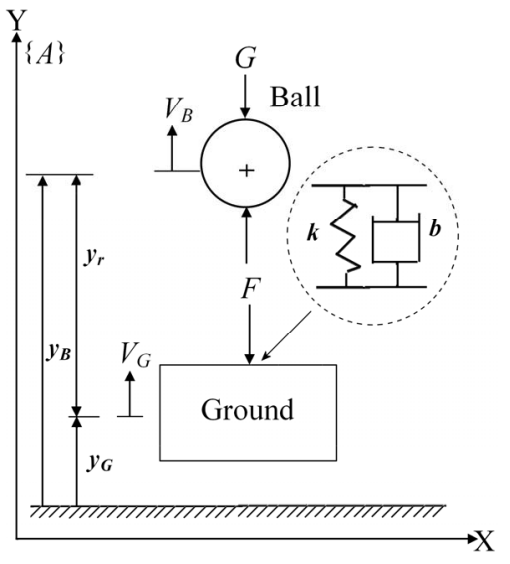

Figure 2. Schematic diagram representing impact between ball and ground.

$$
y_{r}=y_{0}+\int_{0}^{t}\left(V_{B}-V_{G}\right) d \tau .
$$

The general system equation for the contact between ground and ball is given by:

$$
\begin{gathered}
m \frac{d V_{B}}{d t}=-m g+F, \\
b \frac{d y_{G}}{d t}+k y_{G}=-F,
\end{gathered}
$$

where $b$ is the damping coefficient and $k$ is the spring constant used to model impact between the ball and the ground through spring-damper model. $F$ is the Ground Impact force generated due to ball-ground interaction and can be evaluated using Equation (6). Modeling impact between two contact surfaces through springdamper model is categorized as continuous contact dynamics modeling. In this modeling the normal contact force between the contact surfaces is an explicit function of local indentation $\delta$ and its rate [9]. In the case of ball-ground interaction the ground impact force $F$ is a function of $y_{r}$. If $y_{r}>0$ it implies there is no indentation on either the ground or ball and hence $F$ is equal to zero. The ball will be performing ballistic motion under such a situation. The existence or non-existence of the effect of ground-ball contact on the ground impact force can be expressed through Equations (7) and (8). These equations represent switching of values of parameters $b$ and $k$ between zero and certain finite values.

$$
\begin{aligned}
& b=b * \operatorname{swi}\left(0, y_{r}\right), \\
& k=k * \operatorname{swi}\left(0, y_{r}\right),
\end{aligned}
$$

where swi defines a function such as $\operatorname{swi}\left(0, y_{r}\right)=1$, for 0 $\geq y_{r}$, and $\operatorname{swi}\left(0, y_{r}\right)=0$, for $0<y_{r}$.

Hence when $y_{r}>0$ i.e. ball is not in contact with the ground, from Equations (7) and (8) we get $b=k=0$. Hence, ground impact force $F$ is equal to zero. Substituting $F=0$ in Equations (5) and (6), we get

$$
m \frac{d^{2} y_{B}}{d t^{2}}=-m g .
$$

When the ball hits the ground, $y_{r}=0$. From that moment, the ball and the ground move as if a single system. The system equation governing the ball-ground system during contact phase is obtained by combining Equations (5) and (6) and is expressed as,

$$
m \frac{d^{2} y_{G}}{d t^{2}}+b \frac{d y_{G}}{d t}+k y_{G}=-m g
$$

The motion of the ball and ground together as a single system consists of two phases. In the first phase the spring compresses until the ball velocity drops to zero. In the second phase, the spring expands during which the ball starts rebounding. During the entire contact phase the relative displacement of the body with the ground is equal to or less than zero and the detachment occurs when it is positive again.

The bond graph implementation of the impact dynamics between the ball and the ground is shown in Figure 3. Here it is assumed that the ground has zero velocity. Hence the ground velocity junction does not appear in the bond graph.

The parameters used for the simulation are $m_{B}=1.0 \mathrm{~kg}$, spring stiffness $(k)=10^{6} \mathrm{~N} / \mathrm{m}$, damping coefficient $(b)=$ $60 \mathrm{~N}-\mathrm{s} / \mathrm{m}$, Initial height of the ball above the ground $(h)=$ $1.0 \mathrm{~m}$. The corresponding simulation results are presented in Figure 4. It can be noted from Figure 4(a) that the bouncing height over the consecutive hops decays continuously. Figure 4(b) shows the development of contact forces when ball comes in contact with ground. It should be noted that as since the ground impact force model is based on a linear spring damper system the forces are generated whenever there is an indentation/penetration of the_contact surfaces. Figure 4(c) shows the bouncing ball

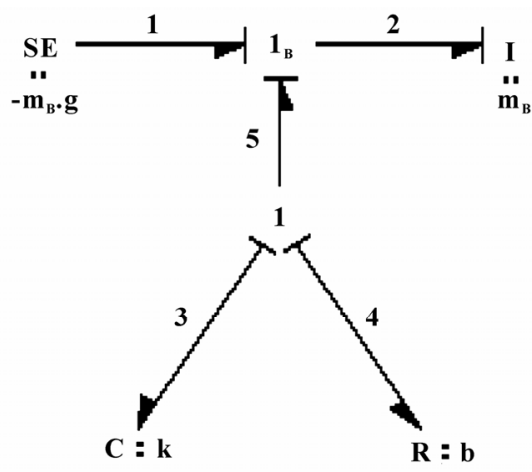

Figure 3. Bond graph representing impact dynamics between ball and ground. 


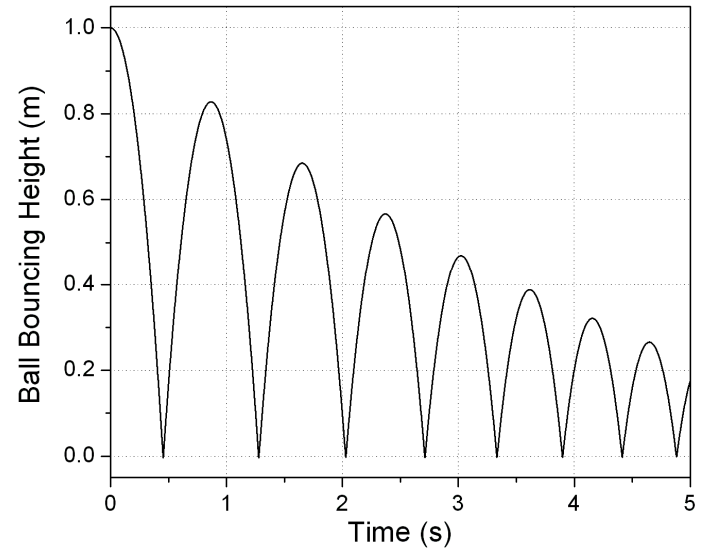

(a)

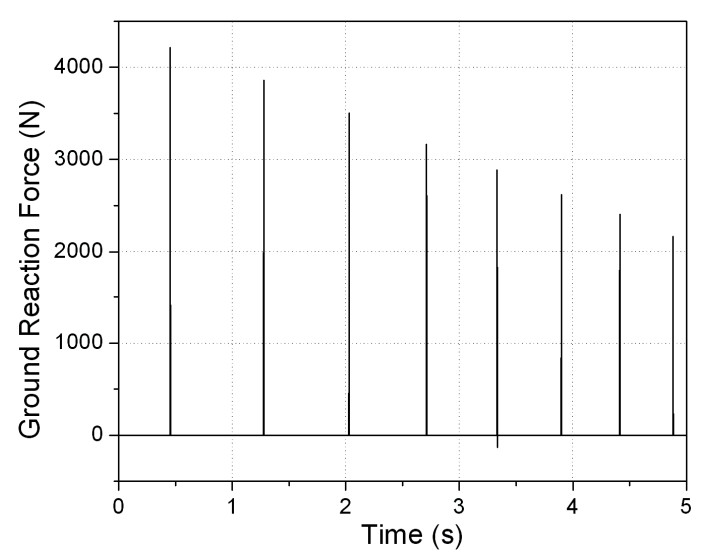

(b)

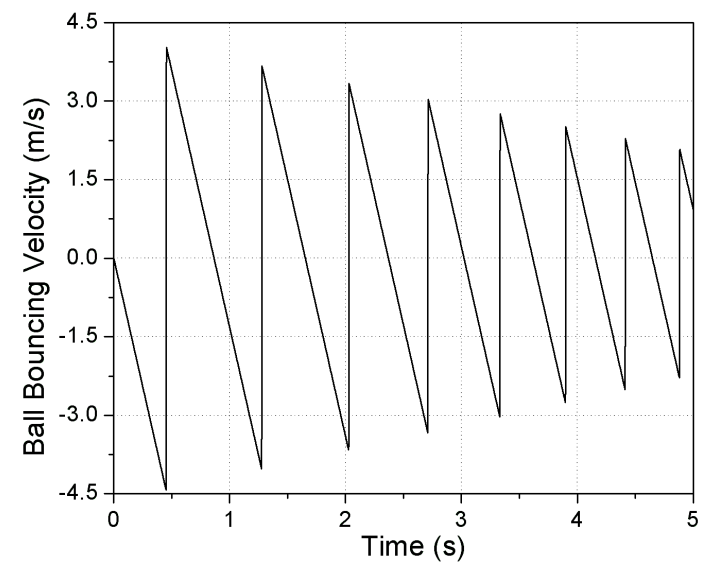

(c)

Figure 4. (a) Ball bouncing height (b) Ground reaction force (c) Ball bouncing velocity.

velocity which decays as time increases. It can be noted from the figure that there is a sudden change in momentum after each successive impact.

Thus the ball bouncing over ground furnishes a simple model of impact between two bodies. It is used in the next section for the modeling of impact of a hopping robot toe with the ground.

\section{Dynamic Modeling of a Hopping Robot}

The hopping robot is modeled as a two mass system based on work carried out by Sato et al. [3]. The first mass is body and the second mass is assumed to be concentrated at its leg tip (toe). The two mass points are connected by a linear motor. A schematic of the hopping robot is shown in Figure 5. The impact dynamics between the robot toe and the ground is modeled on the basis of work presented in the previous section on ball bouncing on the ground. Figure $\mathbf{6}$ shows the bond graph of the hopping robot including the representation of robot toe-ground interaction.

The equations of motion of the hopping robot are given as:

$$
\begin{gathered}
m_{b}{ }^{A} \ddot{z}_{b}=-F_{m}-m_{b} g+F_{b u s}+F_{b l s} \\
m_{t}{ }^{A} \ddot{z}_{t}=F_{m}+F_{e n v}-m_{t} g+F_{t u s}
\end{gathered}
$$

Here $m_{b}$ is the mass of the body; $m_{t}$ is the mass of the toe, $\{A\}$ represents the absolute frame which is located at the ground. $F_{m}$ is the force generated by a linear motor. The interaction between the ground and toe is modeled by linear spring-damper system. $F_{\text {env }}$ is the reaction force from the environment (ground). $K_{g}$ and $R_{g}$ are respectively spring constant and damping coefficient used for modeling the interaction between the toe and ground. $F_{b u s}$ and $F_{b l s}$ are respectively the forces exerted by the

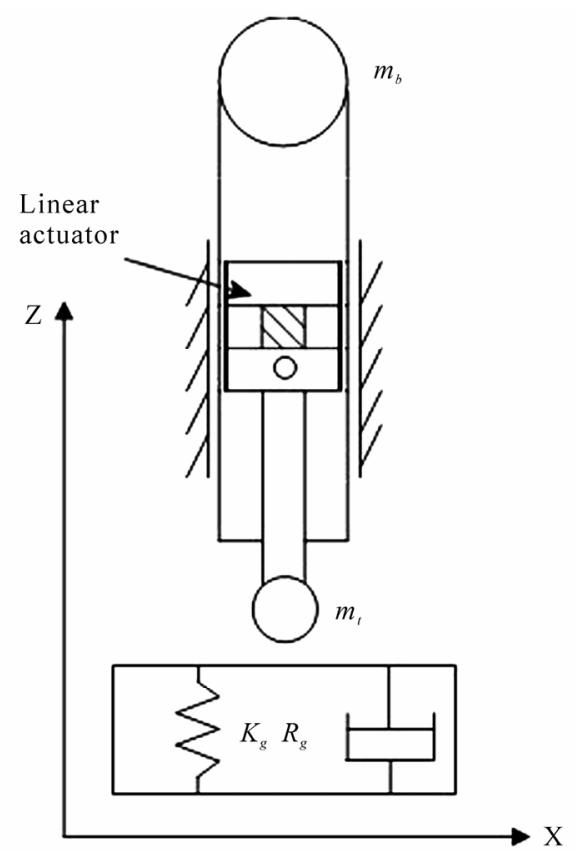

Figure 5. Schematic diagram of a monopod hopping robot. 


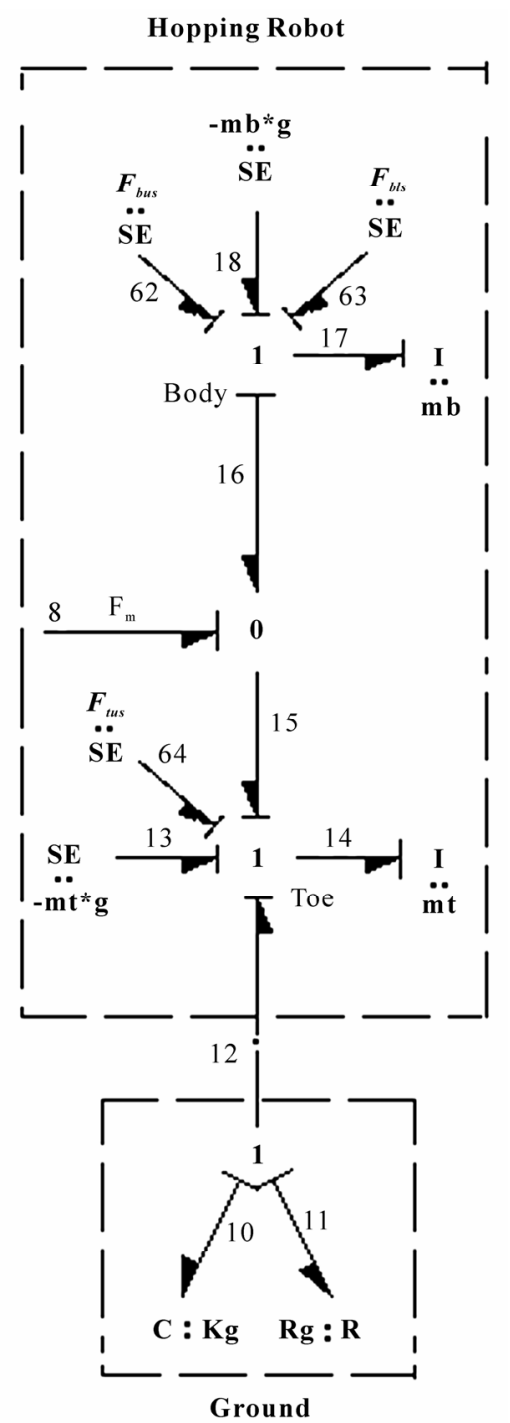

Figure 6. Bond graph model of interaction of a monopod hopping robot with the ground.

upper and lower limiters on the hopping robot body. Similarly $F_{\text {tus }}$ is the force applied by the upper limiter on robot toe. The interaction between the respective limiters and robot body or toe is also modeled by a linear spring-damper system. Corresponding spring constant and damping coefficient values are same as that considered for toe-ground interaction. Considering hard ground, the spring constant and damping coefficient values have been adopted from [3]. The hopping robot system parameters are listed in Table $\mathbf{1}$.

The phases and events of a typical hopping cycle are presented in the Table 2. Figure 7 shows the various phases of the cycle.

In the next section, an impedance controller is designed along with the hopping robot system to attain the desired control of impact forces.
Table 1. Hopping robot parameters.

\begin{tabular}{lcc}
\hline \multicolumn{1}{c}{ Parameters } & $\begin{array}{c}\text { Sym- } \\
\text { bol }\end{array}$ & Value \\
\hline Body mass & $m_{b}$ & $1.3 \mathrm{~kg}$ \\
Leg Mass & $m_{t}$ & $1.0 \mathrm{~kg}$ \\
$\begin{array}{l}\text { Spring coefficient used to model impact } \\
\text { between the leg tip and the ground }\end{array}$ & $K_{g}$ & $10000 \mathrm{~N} / \mathrm{m}$ \\
$\begin{array}{l}\text { Damping coefficient used to model im- } \\
\text { pact between the leg tip and the ground }\end{array}$ & $R_{g}$ & $40 \mathrm{~N}-\mathrm{s} / \mathrm{m}$ \\
$\begin{array}{l}\text { Body Length } \\
\text { Upper limit of body motion }\end{array}$ & $L_{b}$ & $0.5 \mathrm{~m}$ \\
$\begin{array}{l}\text { Lower limit of body motion } \\
\text { Upper limit of Toe motion }\end{array}$ & $Z_{b u s}$ & $1.5 \mathrm{~m}$ \\
\hline
\end{tabular}

Table 2. Hopping cycle.

\begin{tabular}{cc}
\hline Top & Event \\
\hline $\begin{array}{c}\text { Touchdown } \\
\text { Bottom } \\
\text { Liftoff }\end{array}$ & Body CG is highest \\
& Leg Tip leaves ground \\
\hline Stance & Phase \\
\hline Landing & From touchdown to liftoff \\
Thrusting & From touchdown to bottom \\
Aerial & From bottom to liftoff \\
\hline
\end{tabular}

\section{Impedance Control of the Hopping Robot}

The impedance of a system at an interaction port is defined as the ratio between the output effort and the input flow. For applications, demanding a robotic controller to achieve balance between the two characteristics viz. robust trajectory tracking and accommodation of environmental disturbances, the impedance control strategy [6] is best suited.

The impedance control strategy, with regard to the problem under consideration, is based on the body motion compensation. The body motion compensation is so designed that the hopping robot impedance can be modulated to limit the forces of interaction between hopping robot toe and ground. The control paradigm establishes a 


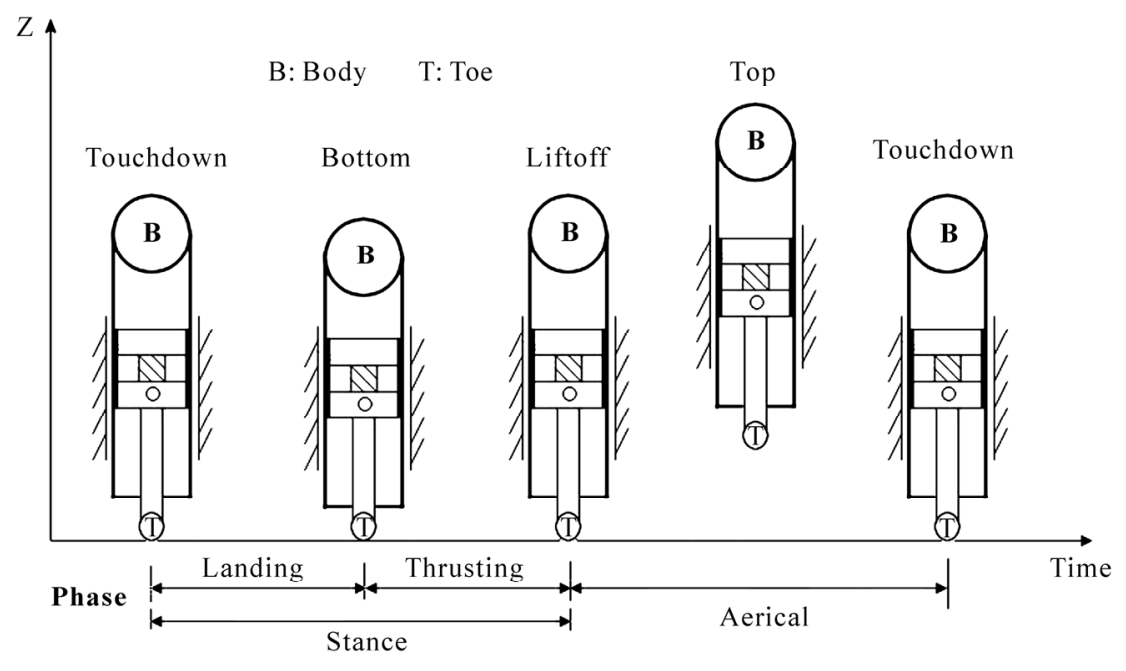

Figure 7. Phases of a hopping cycle.

proper relation between the trajectory controller and the force controller through the manipulation of the impedance. The robot stiffness is made very high during trajectory control, and appropriately modulated during force control. Figure 8 shows the bond graph model of hopping robot with impedance controller.

In this figure $f_{\text {ref }}$ is the reference velocity command for the toe of hopping robot. To incorporate the hopping robot body disturbances in the inertial coordinates, the body velocity is sensed and feedback to the controller. A gain of $\alpha$ shows the feedback compensation. The transfer function between the output flow $F_{t}(s)$ (i.e., the toe velocity) and the input effort $E_{\text {env }}(s)$ (force input from the ground to the toe) represents the admittance $Y_{r o b}(s)$ of the robotic system at the interaction port. The impedance $Z_{r o b}(s)$ is the inverse of the admittance. Admittance at the interaction port can be determined from the bond graph shown in Figure 8.

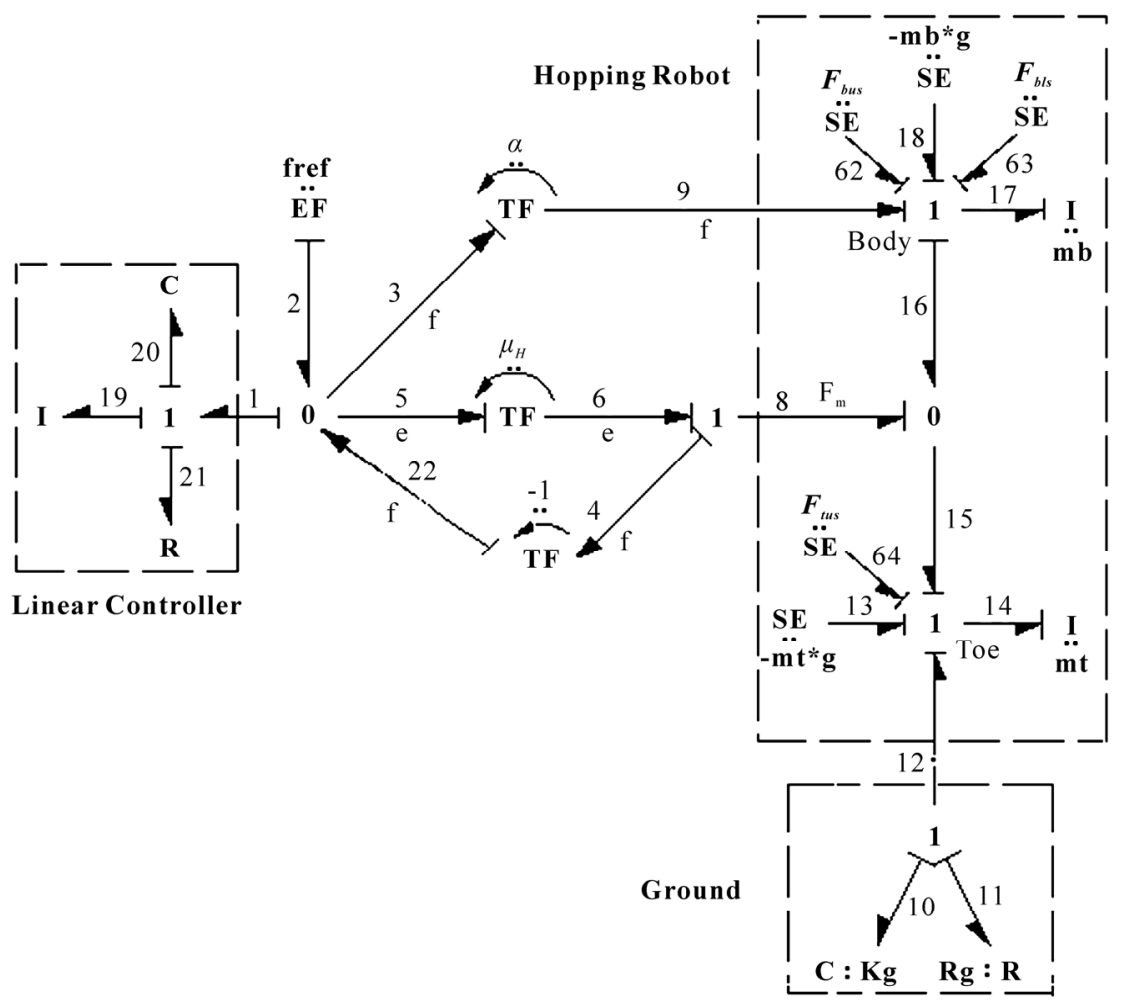

Figure 8. Bond graph of hopping robot with impedance controller. 
The body and toe weights are not considered in this analysis as they can be treated separately as the disturbance force.

Now, applying the constitutive law at junction ' 1 ' corresponding to robot toe, we obtain

$$
e_{12}(t)=e_{14}(t)-e_{15}(t),
$$

Taking Laplace transform on both sides of above expression, we obtain

$$
E_{12}(s)=E_{14}(s)-E_{15}(s),
$$

Transfer function of hopping robot toe can be expressed as

$$
E_{14}(s)=M_{t} s F_{14}(s)=F_{14}(s) / P_{t}(s) .
$$

Constitutive law at junction ' 1 ', corresponding to the controller is given by:

$$
e_{1}(t)=e_{19}(t)+e_{20}(t)+e_{21}(t) .
$$

Taking Laplace transform on both sides, we get

$$
E_{1}(s)=F_{1}(s) C(s)=F_{1}(s)\left(M_{c} s^{2}+R_{c} s+K_{c}\right) / s,(15)
$$

where, $M_{c}, R_{c}$ and $K_{c}$ are respectively the inertia (differential gain), resistance (proportional gain) and stiffness (integral gain) of the controller. From the bond graph, using constituent laws of junctions it can be obtained

$$
E_{15}(s)=\mu_{H} E_{1}(s),
$$

where $\mu_{H}$ is the high feed-forward gain. Next $F_{1}(s)$ can be determined by writing the constituent law at junction ' 0 ' (one which is supplying flow input to controller):

$$
f_{1}(t)-f_{2}(t)+f_{3}(t)+f_{22}(t)=0,
$$

Since reference trajectory is not considered for evaluation of admittance at the interaction port,

$$
f_{1}(t)=-\left[f_{3}(t)+f_{22}(t)\right] .
$$

Substituting corresponding values and taking Laplace transform

$$
F_{1}(s)=\left[(1-\alpha) F_{17}(s)-F_{14}(s)\right] .
$$
(16)

Substituting $F_{1}(s)$ from Equation (18) into Equation

$$
\begin{aligned}
& \qquad E_{15}(s)=\mu_{H} C(s)\left[(1-\alpha) F_{17}(s)-F_{14}(s)\right] . \\
& \text { Also } F_{17}(s)=E_{17}(s) / M_{b} s=P_{b}(s) E_{17}(s),
\end{aligned}
$$

where $P_{b}(s)$ is the transfer function of hopping robot body.

Applying constitutive law at Junction ' 0 ' (corresponding to motor torque $F_{\mathrm{m}}$ ) and at Junction ' 1 ' (corresponding to robot toe), we obtain

$$
E_{17}(s)=-E_{15}(s)=E_{12}(s)-E_{14}(s) .
$$

Substituting $E_{17}(\mathrm{~s})$ from Equation (21) in Equation (20), we get

$$
F_{17}(s)=P_{b}(s)\left[E_{12}(s)-E_{14}(s)\right] .
$$

Combining Equation (13), Equation (14), Equation (19) and Equation (22), we obtain

$$
\begin{aligned}
& E_{12}(s)=\left[F_{14}(s) / P_{t}(s)\right]-\mu_{H} C(s) \\
& {\left[P_{b}(s)(1-\alpha)\left[E_{12}(s)-F_{14}(s) / P_{t}(s)\right]-F_{14}(s)\right]}
\end{aligned}
$$

Simplifying the above equation we get,

$$
\begin{aligned}
& E_{12}(s)\left[1+\mu_{H} C(s)(1-\alpha) P_{b}(s)\right] \\
& =F_{14}(s)\left[\frac{1}{P_{t}(s)}+\mu_{H} C(s)+\mu_{H} C(s)(1-\alpha)\left[\frac{P_{b}(s)}{P_{t}(s)}\right]\right]
\end{aligned}
$$

As since Admittance at the interaction port between hopping robot toe and ground is defined as,

$$
Y_{\text {rob }}(s)=\frac{1}{Z_{r o b}(s)}=\frac{F_{t}(s)}{E_{\text {env }}(s)}=\frac{F_{14}(s)}{E_{12}(s)}
$$

Admittance or impedance at the interaction port is represented as

$$
Y_{r o b}(s)=\frac{P_{t}(s)\left[1+\mu_{H} C(s)(1-\alpha) P_{b}(s)\right]}{\left[1+\mu_{H} C(s) P_{t}(s)+\mu_{H} C(s)(1-\alpha) P_{b}(s)\right]} .
$$

Equation (24) indicates two distinct behavior of the system.

1) When $\alpha=1$, and $\mu_{H}>>1, Y_{r o b}(s)=1 / Z_{r o b}(s)=1 /\left(\mu_{H}\right.$ $C(s)$ ), i.e., toe trajectory is not disturbed by either toe or body inertia so toe can follow the commanded trajectory.

2) When $\alpha<1$, modulation of the impedance to accommodate the interaction forces is possible.

Table 3. Controller parameters.

\begin{tabular}{lcc}
\multicolumn{1}{c}{ Parameters } & Symbol & Value \\
\hline Effort amplifier gain & $\mu_{H}$ & 4 \\
Controller Proportional gain & $r_{c}$ & 100 \\
Controller Derivative gain & $m_{c}$ & 0.001 \\
Controller Integral gain & $K_{c}$ & 2000 \\
Limiting Force & $F_{l i m}$ & $60 \mathrm{~N}$ \\
Gain (Initial Biasing) & $K_{i n i}$ & 0.00 \\
Proportional Gain & $K_{G P}$ & 0.0004 \\
Integral Gain & $K_{G I}$ & 0.0002 \\
\hline
\end{tabular}




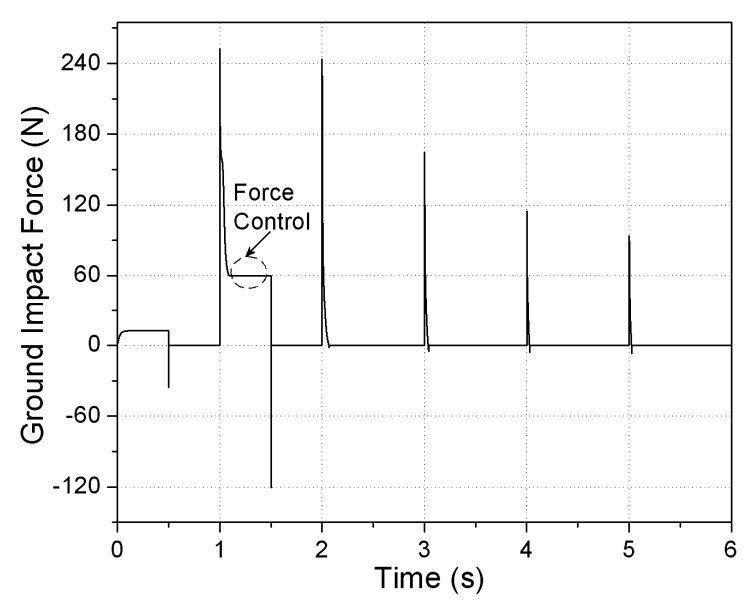

(a)

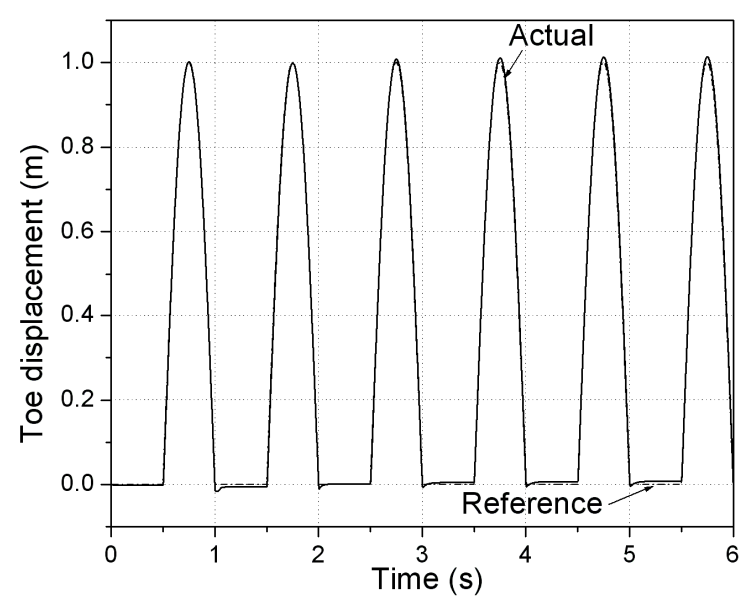

(b)

Figure 9. (a) Ground impact force (b) Toe displacement.

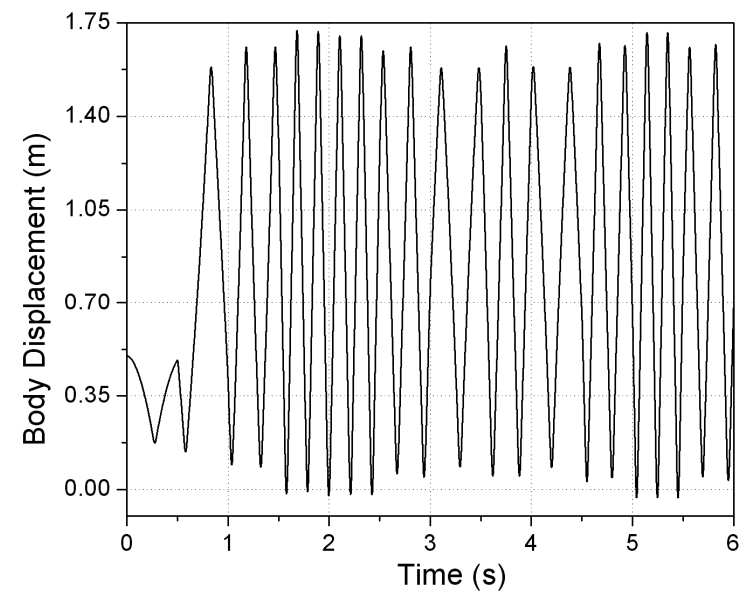

(a)

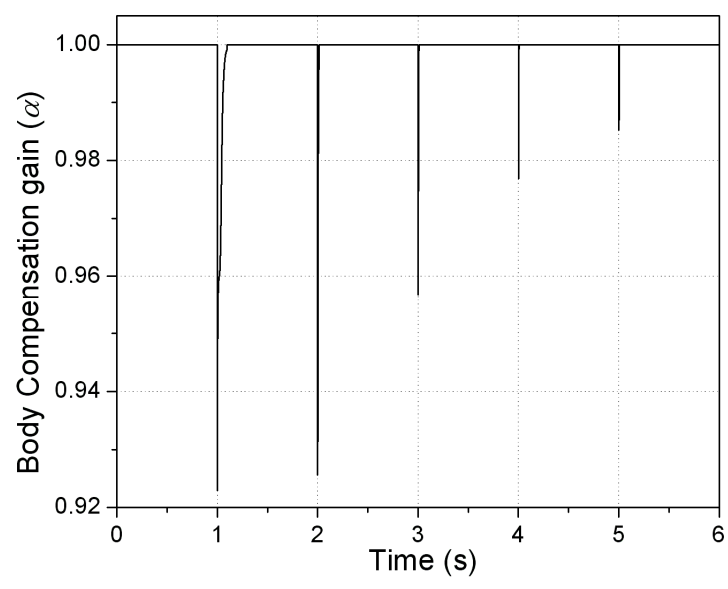

(b)

Figure 10. (a) Body displacement (b) Body compensation gain ( $\alpha$ ).

The heuristic expression for modulation of $\alpha$ is given by,

$$
\begin{aligned}
& \alpha=1-\operatorname{swi}\left(F(t), F_{\lim }\right) \\
& {\left[K_{i n i}+K_{G P}\left(F(t)-F_{\lim }\right)+K_{G I} \int\left(F(t)-F_{\lim }\right) d t\right]}
\end{aligned}
$$

where $F(t)$ is the actual contact force obtained from force sensor; $F_{\text {lim }}$ is the limiting value of the force specified, $K_{i n i}$ is a constant (a bias), $K_{G P}$ is a proportional gain term, and $K_{G I}$ is an integral gain term. Equation (25) represents a proportional-integral control. The swi defines a function such as $s w i(a, b)=1$, for $a \geq b$, and $s w i(a, b)=0$, for $a<b$, where $a, b$ are variables.

The bond graph implementation of the impedance controller with the hopping robot system is shown in Figure 8. Simulation is carried out using SYMBOLS Shakti software. The reference trajectory to be followed by robot toe is taken as a half rectified sine trajectory of am- plitude $2 A$, and is given by Eq. (26) as

$$
y=2 A^{*} \sin (2 \pi t+\pi) * \operatorname{swi}[\sin (2 \pi t+\pi), 0] .
$$

Then the reference velocity command for the toe, is given by,

$$
\dot{y}=4 A \pi \cos (2 \pi t+\pi) * s w i[\sin (2 \pi t+\pi), 0],
$$

At the start of simulation the tip trajectory is initialized to reference trajectory to reduce the initial errors. The parameters values used in simulation are given in Tables $\mathbf{1}$ and $\mathbf{3}$.

The simulation results thus obtained are shown in Figures 9 and 10. Figure 9(a) shows that the force is controlled in the encircled region. At the instant of first impact a very large value of the interaction force i.e. ground impact force (GIF) is generated because toe velocity at the moment of impact is very high. However it is controlled to the specified value of limiting force $\left(F_{\text {Lim }}\right)$ 
equal to $60 \mathrm{~N}$ subsequently. It can be noted that negative peaks of GIF is produced at the beginning of first and second hops. It is due to the change in momentum at the instance of thrusting for next hop by the hopping robot. In subsequent hops the momentum change is of very small value. It is obvious in Figure 9(b) that the robot toe follows reference trajectory very closely. It is interesting to note that the hopping robot is hopping to a constant height continuously for several cycles.

Figure 10(a) shows that the body displacement in the vertical direction is constrained by the upper and lower limiters incorporated into the hopping robot model. Figure 10(b) presents the variation of body compensation gain $(\alpha)$ with respect to time. It varies in order to accommodate the interaction forces generated between the robot toe and ground as shown in Figure 9(a).

\section{Conclusions}

In this paper, impedance control strategy has been used for controlling the impact forces generated during landing phase of the hopping cycle for a monopod robot. Using this strategy the forces generated during landing has been limited to a constant value specified to the impedance controller. Also along with force control very close tracking of leg toe reference trajectory has been attained. This work thus demonstrates the successful realization of impedance control strategy for force control in vertical direction at toe ground interaction point of monopod hopping robot. This model and corresponding analysis can be further extended for developing hopping robot response for multi legged hopping robot, forward running at different velocities.

\section{References}

[1] M. H. Raibert, M. A. Chepponis and H. Brown, "Experiments in Balance with 3D One-Legged Hopping Machine,” International Journal of Robotics Research, Vol. 3, No. 2, 1984, pp. 75-92.

[2] S. H. Hyon and T. Mita, "Development of a Biologically Inspired Hopping Robot-'Kenken',” Proceedings of ICRA'02, IEEE International Conference on Robotics and Automation, Washington, DC, 2002, pp. 3984-3991.

[3] Y. Sato, E. Ohashi and K. Ohnishi, "Impact Force Reduction for Hopping Robot," Proceedings of IEEE 31st Annual Conference of IECON'2005, 6-10 November 2005, pp. 1821-1826.

[4] N. Fujii and K. Ohnishi, "Smooth Transition Method from Compliance Control to Position Control for One Legged Hopping Robot,” Proceedings of IEEE International Conference on Industrial Technology, ICIT 2006, 15-17 December 2006, pp. 164-169.

[5] P. M. Pathak, A. Mukherjee and A. Dasgupta, "Impedance Control of Space Robots using Passive Degrees of Freedom in Controller Domain," ASME Journal of Dynamic Systems, Measurement and Control, Vol. 127, No. 4, 2005, pp. 564-578.

[6] A. Mukherjee, R. Karmarkar and A. K. Samantaray, "Bondgraph in Modeling Simulation and Fault Identification,” I. K. International Publishing House Pvt. Ltd., New Delhi, 2006.

[7] A. Mukherjee, "Users Manual of SYMBOLS Shakti," High-Tech Consultants, S.T.E.P, Indian Institute of Technology, Kharagpur, 2006. http://www.htcinfo.com/

[8] V. Damic and J. Montgomery, Mechatronics by bond graphs: An Object-Oriented Approach to Modeling and Simulation, Springer-Verlag, Berlin, Heidelberg, 2003.

[9] G. Gilardi and I. Sharf, "Literature Survey of Contact Dynamics Modeling”, Mechanism and Machine Theory Vol. 37, No. 10, 2002, pp. 1213-1239. 